\title{
A Survey of the Probability Density Function Control for Stochastic Dynamic Systems
}

\author{
Mifeng Ren \\ College of Information Engineering \\ Taiyuan University of Technology \\ Taiyuan, 030024, China \\ Email: renmifeng@126.com
}

\author{
Qichun Zhang \\ School of Engineering \\ and Sustainable Development \\ De Montfort University \\ Leicester, LE1 9BH, UK \\ Email: qichun.zhang@dmu.ac.uk
}

\author{
Jianhua Zhang \\ State Key Laboratory of \\ Alternate Electrical Power System \\ with Renewable Energy Sources \\ North China Electric Power University \\ Beijing, 102206, China \\ Email: zjh@ncepu.edu.cn
}

\begin{abstract}
Probability density function (PDF) control strategy investigates the controller design approaches in order to to realise a desirable distributions shape control of the random variables for the stochastic processes. Different from the existing stochastic optimisation and control methods, the most important problem of PDF control is to establish the evolution of the PDF expressions of the system variables. Once the relationship between the control input and the output PDF is formulated, the control objective can be described as obtaining the control input signals which would adjust the system output PDFs to follow the pre-specified target PDFs. This paper summarises the recent research results of the PDF control while the controller design approaches can be categorised into three groups: 1) system model-based direct evolution PDF control; 2) model-based distribution-transformation PDF control methods and 3) databased PDF control. In addition, minimum entropy control, PDFbased filter design, fault diagnosis and probabilistic decoupling design are also introduced briefly as extended applications in theory sense.
\end{abstract}

\section{INTRODUCTION}

Since the random noises widely exist in industrial processes, relevant research has been performed to investigate modelling, control and application of stochastic processes. To simplify the system model, we can assume that all the system variables are Gaussian noises. Based on this assumption, many theoretical results and applications have been presented, for example, selfturning control, minimum variance control, linear quadratic Gaussian control and Markov jumping parameter system stochastic control [1], [2]. The design objective of all the mentioned methods only focus on the minimising mean and variance of the system variables. As far as the linear stochastic system with Gaussian random variables is concerned, the shape of the system variable probability density function (PDF) can be fully determined by its mean and variance.

In practice, the system noises in the most of the industrial processes are not necessarily Gaussian; furthermore, the nonlinearity of the systems would also result in non-Gaussian properties even if the investigated system subjected to Gaussian noises. In particular, the mean value and the variance of the system variable cannot be used as a sufficient characterisation tool for the stochastic processes analysis. In other words, the PDF shape of the system variable can be adopted as the more suitable analysis tool to completely characterised the behaviour of a stochastic process. Therefore, a PDF-based control method provides the accuracy and flexibility for the control strategy design with various design requirements.

In the last 20 years, PDF control for stochastic system has been a significant research topic while a lot of relevant results have been presented. To date, PDF-shaping control strategies can generally be classified into: 1) using the evolution of the system PDF which can be described by the partial differential equation (PDE) or inverse function; 2) transforming the PDF by orthogonal decomposition or weight-based neural network; 3) approximating the PDF or joint PDF by data and kernel density estimation (KDE) and 4) PDF optimisation leads to socalled minimum entropy control and its extended applications.

In the following of this survey, we will recall these methods in detail with some theoretical extensions, such as PDF-based filtering, fault diagnosis and probabilistic decoupling, etc. It has been shown that the PDF control methods are significant to industrial process while the practical applications have been introduced in [3], [4]. Therefore, this survey focuses on the theoretical methodologies and omits the industrial background for simplicity.

\section{Model-BASEd DiRect EVOLUTION PDF CONTROL}

PDF control can be restated as minimizing the distance between target PDF and investigated PDF. Therefore, the evolution of the investigated the PDF of the system variable becomes a key task. In other words, we can transform the PDF control to a target tracking description if the PDF can be formulated for the given stochastic dynamic systems. Thus, this section indicates the evolution of the PDF by direct approach and the control strategy is shown by following block diagram.

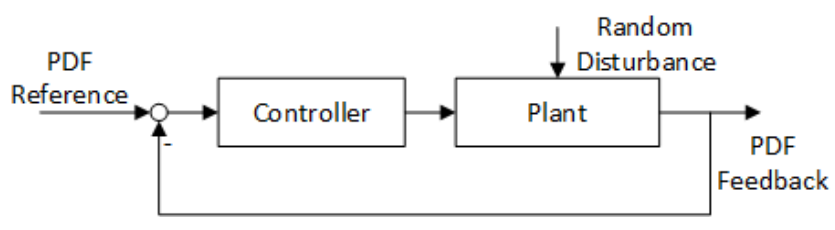

Fig. 1. The block diagram of direct evolution PDF control 


\section{A. Partial differential equation approach}

Generally, Fokker-Planck (FP) equation governs the evolution of the PDF expression for the stochastic process. The continuous time stochastic process has been used to present the stochastic system, which is described by following Ito's stochastic differential equation (SDE)

$$
d X_{t}=b\left(X_{t}, t ; u\right) d t+\sigma\left(X_{t}, t\right) d W_{t}
$$

where $X_{t}=\left(x_{1 t}, \cdots, x_{n t}\right) \in \Omega \subset \mathbb{R}^{n}$ is subjected to the deterministic infinitesimal increments driven by the drift function $b$, and random increments which is given by a independent multi-dimensional Wiener process $d W_{t} \in \mathbb{R}^{m}$.

It is noticed that the variable of a stochastic process can often be fully characterised by the distribution shape. Denote $\gamma(x, t)$ as the PDF for the stochastic process $X_{t}$ with respect to time $t$. Thus, the purpose here is to design a control input signal $u \in \mathbb{R}^{l}$ so that the process evolves towards the prespecified target PDF $\gamma_{d}(x, t)$ with any given initial distribution $\gamma\left(x, t_{0}\right)$. In next essential step, the evolution of the PDF of $X_{t}$ subjected to the dynamics of the stochastic process $X_{t}$ can be recognised and expressed by the following Fokker-Planck (FP) equation [5]:

$$
\begin{aligned}
\frac{\partial \gamma(x, t)}{\partial t} & +\sum_{i=1}^{n} \frac{\partial b_{i}(x, t, u)}{\partial x_{i}} \\
& -\frac{1}{2} \sum_{i, j=1}^{n} \frac{\partial^{2}\left(\left[\sigma(x, t) \sigma^{T}(x, t)\right]_{i j} \gamma(x, t)\right)}{\partial x_{i} \partial x_{j}}=0
\end{aligned}
$$

To approximate the target PDF $\gamma_{d}(x, t)$ as closely as possible, the following cost function was adopted in [6]

$$
J(\gamma, u)=\int\left(\gamma(x, t)-\gamma_{d}(x, t)\right)^{2} d x
$$

Therefore, a consistent framework of formulating an optimal control strategy for stochastic processes can be obtained using the Fokker-Planck equation and the formulated objectives with PDF.

Using the proposed framework, many results of non-linear systems that have exact steady state PDF solutions were obtained in [7], [8]. In particular, optimal control laws can be obtained based upon a receding-horizon model predictive control framework. These control laws minimise the objectives subjected to the given constraint which is governed by the Fokker-Planck equation. PDF-shaping control problems for single dimensional and multidimensional stochastic processes were presented in [9] and [10], respectively. A numerical optimal control was obtained such as Hamiltonian approach [11], minimum principle for infinite dimensional systems [12], etc. Due to restrictions on stability, [13] presented a switching linear controller design while an analytical solution of the PDF was given by the Fokker-Planck-Kolmogorov equation. The FPK-equation-based PDF-shaping control method was also applied to the non-linear filter design [14] and quantum systems [15]. Derived from Kolmogorovs forward equation,
[16] obtained the time evolution of the state PDF with a finite dimensional control input.

The exact transient solutions of Fokker-Plank equations have been obtained only for very special one-dimensional nonlinear stochastic systems.

For some special single dimensional stochastic non-linear systems, the exact transient solutions and exact stationary solutions to Fokker-Plank equations can be obtained. Particularlly, the exact stationary solutions for dissipated multi-degree-offreedom (MDOF) Hamiltonian systems can be divided into 5 classes and all of these solutions can be used to design feedback controller for tracking a pre-specified stationary PDF. Notice that only the stationary solution has been adopted for single dimensional stochastic non-linear system in [17]. Furthermore, [18] proposed an innovative procedure of feedback control design for MDPF stochastic non-linear system using the exact stationary solutions of the dissipated MDOF Hamiltonian systems.

In the above results, the stochastic disturbances are assumed to be Gaussian distribution. However, this assumption is not always the case in practice. Therefore, the generalized FPK (GFPK) equation was used in [19] to deal with the multidimensional non-linear systems with random excitation which is non-Gaussian wide-band stationary. In [20], non-linear systems with Poisson-white-noise are targeted a specified stationary PDF based on the GFPK equation. The procedures to design tracking controllers were summarised.

\section{B. Inverse formula approach}

Since the PDE and SDE are difficult to obtain the analytic solutions, the controller design procedure would be simplified if the evolution of the PDF can be derived without solving PDE and SDE. In order to describe the problem, a general discrete-time input/output model has been given as follows:

$y_{k}=f\left(y_{k-1}, y_{k-2}, \cdots, y_{k-n}, u_{k-d}, u_{k-d-1}, \cdots, u_{k-m}, \omega_{k}\right)$

where $u_{k}$ is the control input, $y_{k}$ stands for the system output, $\omega_{k}$ is a random noise with known PDF $\gamma_{\omega}(x)$ and the dynamics of the system is characterised by $f$. Then, by using the knowledge of the probability theory, the regeneration model between the input/output PDF of the stochastic system output can be formulated as

$$
\gamma\left(y, u_{k}\right)=\gamma_{\omega}\left(f^{-1}\left(\phi_{k}, u_{k}, y\right)\right)\left|\frac{d f^{-1}\left(\phi_{k}, u_{k}, y\right)}{d y}\right|
$$

where $\phi_{k}=\left[y_{k-1}, y_{k-2}, \cdots, u_{k-1}, u_{k-2}, \cdots\right]$ denotes all the historical inputs and outputs of the system which is represented by Equation (4).The numerical solution for the closed-loop control system can be obtained by optimising the following performance index (6).

$$
J=\sum_{k=0}^{k} \int\left(\gamma\left(y, u_{k}\right)-g(y)\right)^{2} d y+u_{k}^{T} R u_{k}
$$

where $g(y), u_{k}$ and $R$ are target PDF, the control input and the weighting matrix, respectively. 
The above control methods can be summarized in [21], where a recursive formula of the conditional output probability density functions evolution was established. However, the stability analysis was not given for the closed-loop system. This resulted in some further developments for the general systems 4. [22] formulated a predictive form for the output PDF. A novel identification method was presented for the stochastic systems with random parameters. In particular, the control input and the system output have been measured to online estimate the unknown PDF of the system parameters while the control input and system output have been used and the output PDF was transformed into a simple algebraic form by the generating functions. this method led to the following development of the scanning least squares algorithm [23]. In addition, the approach has been extended to formulate Joint PDF in [24]. Once the unknown parameters' PDFs were approximated, the control design for output PDF-shaping control could be developed [25].

Note that these direct evolution approaches need to analytically formulate the PDFs of the stochastic dynamic systems, where the initial PDFs are supposed to be known. Also, the computational complexity cannot be ignored.

\section{MODEL-BASED TRANSFORMED EVOLUTION PDF CONTROL}

Without loss of generality, the PDFs of the dynamic stochastic system can be restated in other formats while the control design should be simplified by the equivalent transformation. Based upon the equivalent performance index, the control strategy can be demonstrated by the following block diagram.

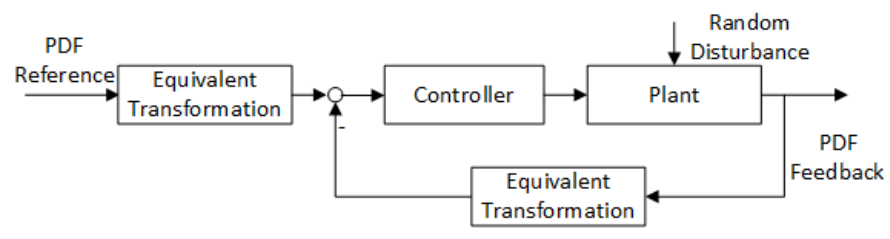

Fig. 2. The block diagram of transformed evolution PDF control

\section{A. Orthogonal decomposition approach}

Iwan and Jensen used the orthogonal decomposition of stochastic functions to deal with some engineering problems with stochastic disturbances in [26], [27], it has been wildly applied to stochastic systems with random parameters [28]. Using this method, the stochastic control problem for systems with randomness can be equivalent to a deterministic one. In order to introduce orthogonal decomposition into PDF-shaping control problem, the following discrete-time stochastic nonlinear process model should be considered:

$$
x_{t+1}=f\left(x_{t}, u_{t}\right)+\omega_{t}
$$

where $x_{t}$ and $u_{t}$ stand for the process state and the manipulated variable, respectively. The function $f$ is analytic in terms of the arguments. The independent and identically random sequence is given as the additive disturbance term $\omega_{t}$ while $t$ stands for integer-valued sampling index. Here, the GramCharlier parametrisation is used to approximate the PDF since it has a close relationship with the moments while the basis functions can be given as follows:

$$
\phi_{i}(x)=(-1)^{i} \frac{\sigma^{i}}{\sqrt{i !}}+\frac{d^{i} N_{\mu, \sigma}(x)}{d x^{i}}
$$

where $N_{\mu, \sigma}(x)$ denotes a Gaussian distribution with standard deviation. The coefficients can be calculated by evaluating the following integrals

$$
c_{i}=\int_{-\infty}^{\infty} g(x) p(x) d x, h_{i}(x)=\frac{\phi_{i}(x)}{N_{\mu, \sigma}(x)}
$$

Thus, the PDF can be approximated by following finite series of GC functions

$$
p(x) \approx \sum_{i=1}^{n} c_{i} h_{i}(x) N_{\mu, \sigma}(x)
$$

The parameters of the controller can be obtained by minimizing the following objective function:

$$
\min J_{G C}=\sum_{i=1}^{n}\left(c_{i}-c_{i, \text { target }}\right)^{2}
$$

Based on the above framework, the problem of targeting a desired stationary PDF was investigated in [13], [29], [30]. Based on Gram-Charlier PDFs, [31] investigated the PDF-shaping control for 1st-order stochastic processes. In [32], the dynamic PDF approximation applications for higherorder processes was presented using the linear control law parametrisations and Gram-Charlier PDF parametrisations. Based upon the parametrisation of the closed-loop stochastic process dynamics and the the corresponding stationary PDF approximation by multivariate Gram-Charlier basis functions, the optimal control design was investigated for discrete-time stochastic processes subjected to a non-quadratic cost function [33].

In the above proposed approaches, by using the GramCharlie expansions as the PDF basis functions, a static feedback control design is presented, then the distribution of the process variable tracks a reference stationary PDF. This technique focused on the stationary state PDF. However it is not a time-evolving PDF. For the non-linear oscillator with random excitation, [29] extended the orthogonal decomposition technique to the stationary response.

The PDF of random variable is re-expressed by a standardized multi-variable orthogonal polynomial set. Using the Galerkin scheme, solving he Fokker-Planck-Kolmogorov (FPK) equation was replaced by a 1st order linear ODE with unknown time-dependent coefficients. Then, uncertainty responses can be obtained with stationary and non-stationary PDFs.

By using the Fokker-Planck forward equation governing the PDF evolution of the process variables, feedback control laws can be developed for continuous-time stochastic non-linear systems so that the closed-loop stochastic system is stationary with the chosen PDF. 


\section{B. Weight-based neural network approach}

The main problem of orthogonal decomposition approach is that the time evolution of the state PDF has not been considered sufficiently. It shows that the implementation of the output PDF control for stochastic system subjected to nonGaussian noise is a challenge topic in the stochastic control filed. In [30], the system output was expressed by a PDF and the control input is formulated with respect to this PDF form. Therefore, obtaining the control input to change the shape of the output PDF following its desired PDF become the purpose of the control design. Notice that the control input is only timerelated. Motivated by the practical problems of paper-making processes, a practically implementable control strategy was originally developed in 1996 [30], which aims at adjusting the output PDF for a class of the stochastic systems with nonGaussian noises.

In [30], B-spline neural network was firstly investigated to approximate the measurable and instantaneous PDFs of the system outputs and the neural networks parameters, such as weights and biases, are dynamically linked to the control input. Thus, the PDF control was converted into the control of the NN parameters.

Suppose that the uniformly bounded random variable $\eta(t) \in$ $[a, b]$ is subjected to the stochastic dynamic system output; $u_{k} \in \mathbb{R}^{m}$ denotes the control input, which is designed to adjust the distribution shape of the system output $y(t) ; \gamma\left(y, u_{k}\right)$ is the PDF of $y(t)$. There are three types of B-spline neural networks to approximate $\gamma\left(y, u_{k}\right)$ [3]:

1) Linear B-spline model (see e.g. [34], [35]):

$$
\gamma\left(y, u_{k}\right)=\sum_{i=1}^{n} B_{i}(y) w_{i}\left(u_{k}\right)+e_{0}, y \in[a, b]
$$

where $w_{i}\left(u_{k}\right)$ stand for the weights of the estimation to the output PDF $\gamma\left(y, u_{k}\right)$ while $B_{i}(y)$ denote the pre-specified basis functions. The main problem of B-spline approximation of PDF is that the trained weights can sometimes be partly negative. It is obvious that this is unacceptable since PDF must be positive.

2) Square root B-spline model(see e.g. [30], [36], [37]): To overcome the disadvantage of this B-spline model, instead of approximating the PDF directly, the square root of the output PDF should be estimated using the following approach.

$$
\sqrt{\gamma\left(y, u_{k}\right)}=\sum_{i=1}^{n} B_{i}(y) w_{i}\left(u_{k}\right)+e_{0}, y \in[a, b]
$$

3) Rational B-spline model: In order to consider the condition $\int \gamma\left(y, u_{k}\right) d y=1$, the weights of the B-spline $\mathrm{NN}$ are constrained. Therefore, the modified model is used as follows:

$$
\gamma\left(y, u_{k}\right)=\frac{\sum_{i=1}^{n} B_{i}(y) w_{i}\left(u_{k}\right)}{\sum_{i=1}^{n} b_{i} w_{i}\left(u_{k}\right)}+e_{0}, y \in[a, b]
$$

where $b_{i}=\int_{a}^{b} B_{i}(y) d y>0$.

Since the integration of $\gamma\left(y, u_{k}\right)$ over its definition domain $[a, b]$ must be 1 , it has been shown that only $n-1$ weights are independent. We can further denote $V_{k}$ as the vector of independent weights, then the dynamic of the system can be rewritten as

$$
V_{k+1}=f\left(V_{k}, u_{k}\right)
$$

where $f\left(V_{k}, u_{k}\right)$ is a vector function representing the dynamics of the vector-valued $\mathrm{NN}$ weights and the control input. Therefore, Equations (12)-(15) supply the general structure of modelling the stochastic distribution systems, where the control input is time-varying and the model output is the output PDF. Then, the optimal control algorithm should be obtained by searching the optimum of the performance index (6) minimum.

In the above framework, a lot of controller designs have been obtained to adjust the shape of the output PDF. [30] established a linear feedback controller where the control law was presented using the measured output PDFs and the input signal with linear dynamics. After that, this method was extended for the stochastic non-linear systems [38]. In addition, [30] analysed the robustness of the closed-loop systems. In [30], a novel scanning, recursive parameter approximation algorithm was presented to estimate the linear parameters in model (15) using the unknown $f\left(V_{k}, u_{k}\right)$. Based upon the system dimensions, the multi-layer perceptions (MLPs) [39], [40] and radial basis functions (RBFs) [41]-[43] can also be used to estimate the output PDFs.

Although the PDF-shaping problem can be well solved by using the above neural networks model, there are several problems of this method, such as 1) For the dynamics of the weighting vector, the shape of the PDFs cannot be changed if only linear models are established; 2) the high computational load is the key problem of the numerical solution and the performance is difficult to meet the requirements including the stability and the robustness of the closed-loop realization. For the practical system implementation, the fixed-structure controller has been developed to overcome the mentioned shortcomings. In particular, PID controller has been presented in [44]-[46], where the parameters of the controller can be obtained using linear matrix inequalities (LMIs). Using the fixed-structure controller design, the total dimension of the parameters can be minimised and off-line design would simplify the algorithm with stability analysis which can be considered as the advantages. Notice that a synthesis and integrated analysis framework can be built up with LMI techniques.

Although different types of neural networks can be used to replace $\mathrm{B}$-spline $\mathrm{NN}$ as theoretical extensions, the main problem with the described B-spline approach is that the direct physical meaning of the controller design model is not convincing. Meanwhile, the complicated shape of the output PDF leads to a complex neural networks which also results in the high dimensional dynamics between the control input and the weighting vector (see model (15)).

\section{DATA-BASED PDF CONTROL}

In practice, it is difficult to establish system dynamic models represented practical industrial processes with non- 
Gaussian noises, therefore, it dose not make sense to use the mentioned model-based approach for these processes which leads to model-free stochastic distribution control strategy. The block diagram is shown below where the indirect databased approach is given if the model identification procedure is included otherwise the PDF can be approximated by data directly using KDE which called direct data-based approach or data-driven approach.

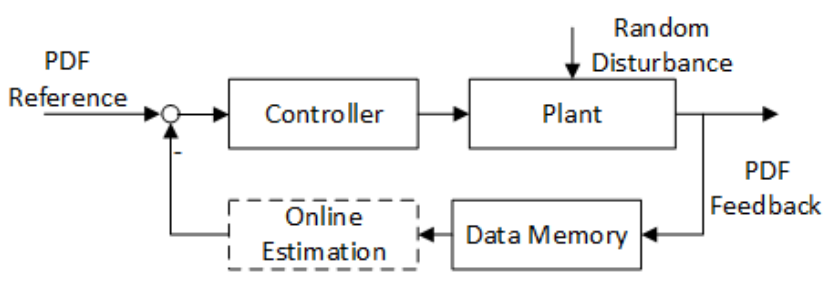

Fig. 3. The block diagram of data-based PDF control

As mentioned in previous section, neural networks were established for both modelling and control of the stochastic non-linear non-Gaussian systems in [45], [47], [48]. Using the square-root B-spline NN estimation to the measured output PDF, the problem has been converted as the dynamic weights tracking. Fix-structure NN with unknown parameters were employed to describe the dynamics between the weighting vector and the control input. [49] presented a dynamic neural network for modelling and control the investigated plant where the randomness of the system output has been attenuated by minimum entropy control scheme with an iterative learning control (ILC) basis. The above control strategy is usually called data-driven SDC or model-free SDC. In fact, we note that these control algorithms were designed on the basis of the hidden or implicit system modelling. In other words, they are not actually model-free.

Based on the MEE criterion, an optimal control method has been obtained for the semiconductor processes subjected to non-Gaussian noise in [50]. Parzen window technique [51] was introduced to estimate the PDF and quadratic Rényi entropy of the tracking error, and the optimised control input was directly designed based on the sample data of the tracking error without approximating the dynamic system. Motivated by information theory, [52] investigated the adaptive control problem for nonGaussian stochastic systems using single neuron. The control algorithm was developed with the neuron weights training under the generalized MEE principle while the cost function can be estimated by the Parzen windowing technique. The joint PDF can also be controlled by data-driven approach [53]. Moreover, [54], [55] proposed the nueral PID controller for a class of non-linear non-Gaussian system by minimising the entropy of the system error. The advantage of this date-based method is that the control algorithms are developed in the databased framework, while the accuracy of the system model is not essential since the accurate model is difficult to obtain. However, the model is still needed when analysing stochastic systems.

\section{EXTENSIONS: MINIMUM ENTROPY CONTROL, FILTERING, FAULT DIAGNOSIS AND PROBABILISTIC DECOUPLING}

In the previous sections, the control algorithms have been presented where we suppose to the known target PDF. However, the target PDF sometimes is not available. In this case, the performance criterion can be selected as the minimum entropy, such as [56]-[58]. In particular, the performance criterion is defined as follows:

$$
J=-\sum_{k=0}^{k} \int \gamma\left(y, u_{k}\right) \log \left(\gamma\left(y, u_{k}\right)\right) d y+u_{k}^{T} R u_{k}
$$

or defining $e=r-y$ with $r$ is the set point, we have

$$
J=\int \gamma\left(e, u_{k}\right) \log \left(\gamma\left(e, u_{k}\right)\right) d e+u_{k}^{T} R u_{k}
$$

while the first term denotes the entropy of the system output in (16) and tracking error in (17), respectively.

For measurable output PDFs, the B-spline approach is used to re-express the measured output PDF and the system dynamics is described by a set of differential or difference equations which link the B-spline weighting vector to the control input (see e.g. [59]) and Equation (5) should be considered to further formulate the relationship of the system output PDF and the control input even if the PDF is unmensurable. [60] presented a recursive optimization solution based on MEE control which guaranteed the local stability for the closed-loop system. The result in [60] was extended to the a stochastic system model with two inputs and two outputs [61]. As a summary, the MEE principle has been widely used for control and optimisation of the stochastic non-Gaussian systems (see e.g. [62], [63]).

Filtering design and fault diagnosis are two important problems in non-linear and non-Gaussian systems. Recently, the SDC concept has been applied to investigate these problems [42], [48], [62], [64]-[67].

Moreover, a novel decoupling control strategy entitled probabilistic decoupling was presented for stochastic dynamic systems [68], [69] while the couplings among the system outputs have been quantized in terms of the output PDFs. In order to minimise the couplings in probability sense, the distance between the joint PDF and the product of output marginal PDFs has been considered as performance index. Furthermore, the mutual information criterion can be used as an extended minimum entropy performance index to achieve the probabilistic decoupling [70]. Based on the similar structure, the performance enhancement of the stochastic dynamic system is also investigated in [71] using PDF optimisation.

\section{CONCLUSION}

This survey reports the developments of the PDF control in four aspects: direct evolution, transformed evolution, databased approximation and PDF control applications. All the methods in this category can be included as stochastic distribution control with the similar structure [13], basically, (i) briefing the non-linearity, parametrisation for stochastic processes, (ii) formulating an approximate or parametrised 
PDF expression and (iii) minimising an appropriate criterion to characterise the distance between output PDFs and desired PDFs.

All the mentioned methods have their own benefits and shortcomings, in particular, the direct evolution approach needs to derive the complex analytical formula and the stability of the data-driven framework should be further analysed. Meanwhile, the orthogonal decomposition technique dose not take time evolution of PDF into account and neural network modelling does not indicate the physical meaning of the PDF evolution. Since the extended applications have also been discussed in this paper, the PDF control would affect other research fields in the future. In other words, there are still many challenges for this research topic.

\section{ACKNOWLEDGEMENTS}

This work was supported in part by the DMU HEIF'18 project, in part by the Natural Science Foundation of Shanxi Province under Grant 201701D221112, and in part by the National Natural Science Foundation of China under Grants 61503271 and 61603136, these are acknowledged.

\section{REFERENCES}

[1] K. J. Astrom, Introduction to Stochastic Control Theory. Elsevier, 1971, vol. 70.

[2] G. C. Goodwin and K. S. Sin, Adaptive filtering prediction and control. Courier Corporation, 2014.

[3] A. Wang, H. Wang, and L. Guo, "Recent advances on stochastic distribution control: Probability density function control," in Control and Decision Conference, 2009. CCDC'09. Chinese. IEEE, 2009, pp. xxxv-xli.

[4] H. Yue and H. Wang, "Recent developments in stochastic distribution control/a review," Measurement and Control, vol. 36, no. 7, pp. 209-215, 2003.

[5] P. Whittle, Probability via expectation. Springer Science \& Business Media, 2012.

[6] L. Crespo and J. Sun, "Nonlinear stochastic control via stationary probability density functions," in American Control Conference, 2002. Proceedings of the 2002, vol. 3. IEEE, 2002, pp. 2029-2034.

[7] O. Elbeyli and J. Sun, "A stochastic averaging approach for feedback control design of nonlinear systems under random excitations," Journal of vibration and acoustics, vol. 124, no. 4, pp. 561-565, 2002.

[8] L. Socha and M. Blachuta, "Application of linearization methods with probability density criteria in control problems," in American Control Conference, 2000. Proceedings of the 2000, vol. 4. IEEE, 2000, pp. 2775-2779.

[9] M. Annunziato and A. Borzì, "Optimal control of probability density functions of stochastic processes," Mathematical Modelling and Analysis, vol. 15, no. 4, pp. 393-407, 2010.

[10] —, "A fokker-planck control framework for multidimensional stochastic processes," Journal of Computational and Applied Mathematics, vol. 237, no. 1, pp. 487-507, 2013.

[11] A. Palmer and D. Milutinović, "A hamiltonian approach using partial differential equations for open-loop stochastic optimal control," in American Control Conference (ACC), 2011. IEEE, 2011, pp. 2056-2061.

[12] H. O. Fattorini, Infinite dimensional optimization and control theory. Cambridge University Press, 1999, vol. 54.

[13] B. Pigeon, M. Perrier, and B. Srinivasan, "Shaping probability density functions using a switching linear controller," Journal of Process Control, vol. 21, no. 6, pp. 901-908, 2011.

[14] S. Challa and Y. Bar-Shalom, "Nonlinear filter design using fokkerplanck-kolmogorov probability density evolutions," IEEE Transactions on Aerospace and Electronic Systems, vol. 36, no. 1, pp. 309-315, 2000.

[15] M. Annunziato and A. Borzi, "On a fokker-planck approach to control open quantum systems," in Nonlinear Dynamics of Electronic Systems, Proceedings of NDES 2012. VDE, 2012, pp. 1-5.
[16] K. Ohsumi and T. Ohtsuka, "Particle model predictive control for probability density functions," IFAC Proceedings Volumes, vol. 44, no. 1, pp. 7993-7998, 2011.

[17] M. Forbes, M. Guay, and J. F. Forbes, "Probabilistic control design for continuous-time stochastic nonlinear systems: a pdf-shaping approach," in Intelligent Control, 2004. Proceedings of the 2004 IEEE International Symposium on. IEEE, 2004, pp. 132-136.

[18] C. Zhu and W. Zhu, "Feedback control of nonlinear stochastic systems for targeting a specified stationary probability density," Automatica, vol. 47, no. 3, pp. 539-544, 2011.

[19] Y. Zeng and W. Zhu, "Stochastic averaging of n-dimensional nonlinear dynamical systems subject to non-gaussian wide-band random excitations," International Journal of Non-Linear Mechanics, vol. 45, no. 5, pp. 572-586, 2010.

[20] C. Zhu and W. Zhu, "Control of stationary probability density of nonlinear systems subject to poisson white noise excitation," Journal of Vibration and Control, vol. 20, no. 3, pp. 323-329, 2014.

[21] H. Wang, "Control of conditional output probability density functions for general nonlinear and non-gaussian dynamic stochastic systems," IEE Proceedings-Control Theory and Applications, vol. 150, no. 1, pp. $55-60,2003$.

[22] L. Guo, H. Wang, and A. Wang, "Optimal probability density function control for narmax stochastic systems," Automatica, vol. 44, no. 7, pp. 1904-1911, 2008

[23] H. Wang, A. Wang, and Y. Wang, "Online estimation algorithm for the unknown probability density functions of random parameters in autoregression and exogenous stochastic systems," IEE Proceedings-Control Theory and Applications, vol. 153, no. 4, pp. 462-468, 2006.

[24] L. Yin and L. Guo, "Joint stochastic distribution tracking control for multivariate descriptor systems with non-gaussian variables," International Journal of Systems Science, vol. 43, no. 1, pp. 192-200, 2012.

[25] Y. Wang and H. Wang, "Output pdfs control for linear stochastic systems with arbitrarily bounded random parameters: a new application of the laplace transform," in American Control Conference, 2002. Proceedings of the 2002, vol. 5. IEEE, 2002, pp. 4262-4267.

[26] H. Jensen and W. Iwan, "Response variability in structural dynamics," Earthquake engineering \& structural dynamics, vol. 20, no. 10, pp. 949959, 1991.

[27] W. Iwan and H. Jensen, "On the dynamic response of continuous systems including model uncertainty," Journal of applied mechanics, vol. 60, no. 2, pp. 484-490, 1993.

[28] T. Fang, X. Leng, and C. Song, "Chebyshev polynomial approximation for dynamical response problem of random system," Journal of Sound and Vibration, vol. 266, no. 1, pp. 198-206, 2003.

[29] X. Zhang, Y. Zhang, M. Pandey, and Y. Zhao, "Probability density function for stochastic response of non-linear oscillation system under random excitation," International Journal of Non-Linear Mechanics, vol. 45, no. 8, pp. 800-808, 2010.

[30] H. Wang, Bounded dynamic stochastic systems: modelling and control. Springer Science \& Business Media, 2012.

[31] M. Forbes, M. Guay, and J. Forbes, "Control design for first-order processes: shaping the probability density of the process state," Journal of process control, vol. 14, no. 4, pp. 399-410, 2004.

[32] M. G. Forbes, J. F. Forbes, and M. Guay, "Regulatory control design for stochastic processes: shaping the probability density function," in American Control Conference, 2003. Proceedings of the 2003, vol. 5. IEEE, 2003, pp. 3998-4003.

[33] _ - "Control design for discrete-time stochastic nonlinear processes with a nonquadratic performance objective," in Decision and Control, 2003. Proceedings. 42nd IEEE Conference on, vol. 4. IEEE, 2003, pp. $4243-4248$.

[34] H. Wang, J. Zhang, and H. Yue, "Periodic learning of b-spline models for output pdf control: application to mwd control," in American Control Conference, 2005. Proceedings of the 2005. IEEE, 2005, pp. 955-960.

[35] X. Sun, H. Yue, and H. Wang, "Modelling and control of the flame temperature distribution using probability density function shaping," Transactions of the Institute of Measurement and Control, vol. 28, no. 5, pp. 401-428, 2006.

[36] J. Zhang, W. Wang, J. Zhang, and G. Hou, "Probability density function predictive control based on the rational square-root b-spline model," in Control and Decision Conference, 2009. CCDC'09. Chinese. IEEE, 2009, pp. 4669-4672.

[37] H. Chen and H. Wang, "Pdf control of stochastic parameter system using linear matrix inequalities," ACTA Automatica Sinica, vol. 33, no. 11, pp. 1216-1220, 2007. 
[38] H. Wang, P. Kabore, and H. Baki, "Lyapunov-based controller design for bounded dynamic stochastic distribution control," IEE ProceedingsControl Theory and Applications, vol. 148, no. 3, pp. 245-250, 2001.

[39] Y. Zhang, L. Guo, H. Yu, and K. Zhao, "Fault tolerant control based on stochastic distributions via mlp neural networks," Neurocomputing, vol. 70, no. 4-6, pp. 867-874, 2007.

[40] W. Wang, Y. L. Xiong, H. Wang, and H. Yue, "Mimo probability density function control using simple log-mlp neural networks," in International Conference on Intelligent Robotics and Applications. Springer, 2008, pp. $820-828$.

[41] Z. Skaf, H. Wang, and L. Guo, "Fault tolerant control based on stochastic distribution via rbf neural networks," Journal of Systems Engineering and Electronics, vol. 22, no. 1, pp. 63-69, 2011.

[42] Q. Yi, L. Zhan-Ming, and L. Er-Chao, "Fault detection and diagnosis for non-gaussian stochastic distribution systems with time delays via rbf neural networks," ISA transactions, vol. 51, no. 6, pp. 786-791, 2012.

[43] P. Afshar, M. Brown, and H. Wang, "Gradient descent optimisation for ilc-based stochastic distribution control," in Control and Automation, 2009. ICCA 2009. IEEE International Conference on. IEEE, 2009, pp. 1134-1139.

[44] L. Guo and H. Wang, "Stochastic distribution control: Ma-trix inequality approach," 2009.

[45] Y. Yi, L. Guo, and H. Wang, "Constrained pi tracking control for output probability distributions based on two-step neural networks," IEEE Transactions on Circuits and Systems I: Regular Papers, vol. 56 no. 7, pp. 1416-1426, 2009.

[46] Z. Skaf, A. H. Al-Bayati, and H. Wang, "An adaptive pi controller for non-gaussian stochastic systems," in Intelligent Control and Automation (WCICA), 2010 8th World Congress on. IEEE, 2010, pp. 832-837.

[47] L. Guo, Y. Yi, and H. Wang, "Statistic information tracking of nongaussian systems: A data-driven control framework based on adaptive nn modeling," in Networking, Sensing and Control, 2009. ICNSC'09. International Conference on. IEEE, 2009, pp. 170-175.

[48] Y. Zhang, Y. Liu, and L. Guo, "A data-driven based adaptive fault diagnosis scheme for nonlinear stochastic distribution systems via 2step neural networks and descriptor model," in Intelligent Control and Automation (WCICA), 2012 10th World Congress on. IEEE, 2012, pp. 3311-3315.

[49] P. Afshar, H. Wang, and T. Chai, "An ilc-based adaptive control for general stochastic systems with strictly decreasing entropy," IEEE transactions on neural networks, vol. 20, no. 3, pp. 471-482, 2009.

[50] J. Zhang, C.-C. Chu, J. Munoz, and J. Chen, "Minimum entropy based run-to-run control for semiconductor processes with uncertain metrology delay," Journal of Process Control, vol. 19, no. 10, pp. 1688-1697, 2009.

[51] D. Erdogmus, "Information theoretic learning: Renyi's entropy and its applications to adaptive system training," Ph.D. dissertation, University of Florida Gainesville, Florida, 2002.

[52] M. Ren, J. Zhang, M. Jiang, Y. Tian, and G. Hou, "Statistical information based single neuron adaptive control for non-gaussian stochastic systems," Entropy, vol. 14, no. 7, pp. 1154-1164, 2012.

[53] L. Yin, H. Zhang, and L. Guo, "Data driven output joint probability density function control for multivariate non-linear non-gaussian systems," IET Control Theory \& Applications, vol. 9, no. 18, pp. 2697-2703, 2015.

[54] J. Zhang, F. Zhang, M. Ren, G. Hou, and F. Fang, "Cascade control of superheated steam temperature with neuro-pid controller," ISA transactions, vol. 51, no. 6, pp. 778-785, 2012.

[55] J. Zhang, F. Zhang, and H. Wang, "Neural pid control strategy for superheated steam temperature based on minimum entropy," in Advanced
Mechatronic Systems (ICAMechS), 2011 International Conference on. IEEE, 2011, pp. 524-528.

[56] Y. Yi, T. Li, L. Guo, and H. Wang, "Statistic tracking strategy for nongaussian systems based on pid controller structure and lmi approach," Dynamics of Continuous, Discrete and Impulsive Systems-B, vol. 15, pp. 859-872, 2008.

[57] P. Afshar, A. Nobakhti, and H. Wang, "Probabilistic quality control in non-gaussian process control applications," in Automation and Logistics, 2009. ICAL'09. IEEE International Conference on. IEEE, 2009, pp. 498-503.

[58] P. Afshar, A. Nobakhti, H. Wang, and T. Chai, "Multi-objective minimum entropy controller design for stochastic processes," in American Control Conference (ACC), 2010. IEEE, 2010, pp. 355-360.

[59] Y. Yi, T. Li, and L. Guo, "Statistic tracking control for non-gaussian systems using ts fuzzy model," IFAC Proceedings Volumes, vol. 41, no. 2, pp. 11564-11569, 2008.

[60] H. Yue and H. Wang, "Minimum entropy control of closed-loop tracking errors for dynamic stochastic systems," IEEE Transactions on Automatic Control, vol. 48, no. 1, pp. 118-122, 2003.

[61] J. Zhang, M. Ren, and H. Wang, "Minimum entropy control for nonlinear and non-gaussian two-input and two-output dynamic stochastic systems," IET Control Theory \& Applications, vol. 6, no. 15, pp. 2434 2441, 2012.

[62] J. Zhang, L. Du, M. Ren, and G. Hou, "Minimum error entropy filter for fault detection of networked control systems," Entropy, vol. 14, no. 3, pp. 505-516, 2012.

[63] M. Ren, J. Zhang, J. Zhang, and G. Hou, "Minimum entropy incremental control for nonlinear stochastic systems with non-gaussian disturbances and uncertain parameters," in American Control Conference (ACC), 2012. IEEE, 2012, pp. 197-201.

[64] L. Yao, J. Qin, H. Wang, and B. Jiang, "Design of new fault diagnosis and fault tolerant control scheme for non-gaussian singular stochastic distribution systems," Automatica, vol. 48, no. 9, pp. 2305-2313, 2012.

[65] Y. Ren and H. Wang, "Fault tolerant control for sequentially connected stochastic distribution continuous systems," in Modelling, Identification and Control (ICMIC), The 2010 International Conference on. IEEE, 2010, pp. 471-476.

[66] L. Yin, P. Zhu, and T. Li, "Fault detection and diagnosis for delay-rangedependent stochastic systems using output pdfs," International Journal of Control, Automation and Systems, vol. 15, no. 4, pp. 1701-1709, 2017.

[67] L. Yin and L. Zhou, "Function based fault detection for uncertain multivariate nonlinear non-gaussian stochastic systems using entropy optimization principle," Entropy, vol. 15, no. 1, pp. 32-52, 2012.

[68] Q. Zhang, J. Zhou, H. Wang, and T. Chai, "Minimized coupling in probability sense for a class of multivariate dynamic stochastic control systems," in Decision and Control (CDC), 2015 IEEE 54th Annual Conference on. IEEE, 2015, pp. 1846-1851.

[69] Q. Zhang and X. Yin, "Observer-based parametric decoupling controller design for a class of multi-variable non-linear uncertain systems," Systems Science \& Control Engineering, vol. 6, no. 1, pp. 258-267, 2018.

[70] Q. Zhang and A. Wang, "Decoupling control in statistical sense: minimised mutual information algorithm," International Journal of Advanced Mechatronic Systems, vol. 7, no. 2, pp. 61-70, 2016.

[71] Y. Zhou, Q. Zhang, H. Wang, P. Zhou, and T. Chai, "Ekf-based enhanced performance controller design for nonlinear stochastic systems," IEEE Transactions on Automatic Control, vol. 63, no. 4, pp. 1155-1162, 2018. 